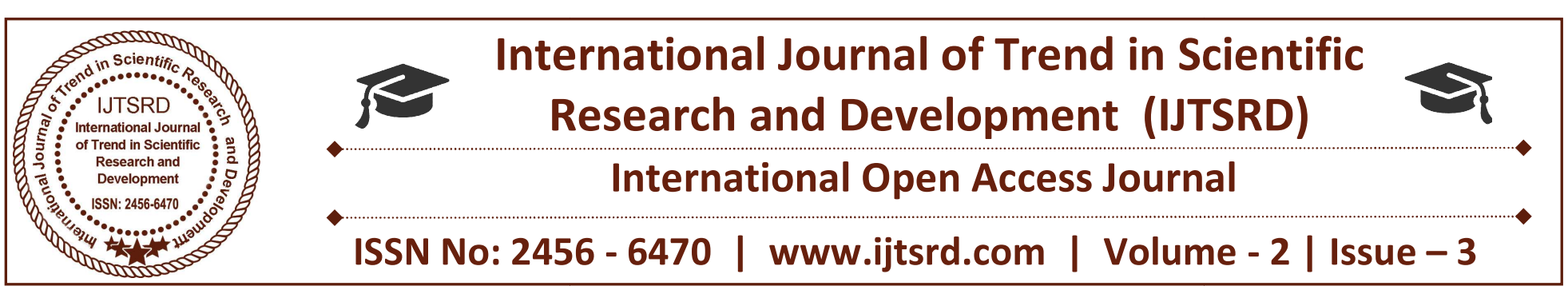

\title{
Club Management System using Laravel Framework
}

\author{
Sindhu Madhukar ${ }^{1}$, Suhas $\mathbf{G}^{2}$ \\ ${ }^{1}$ M.Tech Student, ${ }^{2}$ Assistant Professor, \\ Department of Computer Science and Engineering, \\ Maharaja Institute of Technology, Mysore, Karnataka, India
}

\begin{abstract}
CMS (Club Management system) is an integrated management system handling the common functions of enrolment, fulfillment, member/club/promotion information support, claims, billing and standard reporting via additional sub system and this project is used to give support to the client. In this project we are using a Radio Frequency Identification (RFID) and Thermal printers. Radio Frequency Identification (RFID) provides a smooth payment and secure access. The CMS system contains different clubs like Bar Club, providing services to customers. Legal Care application being a part of CMS does enrolment, fulfillment and billing for customers through different partners, and the application is built in the laravel framework.
\end{abstract}

\section{Keywords: Club Management system, RFID Readers}

\section{INTRODUCTION}

Club Management System (CMS) is designed to provide a club with everything that it need to manage all its activities through a single application. Furthermore the system can be extended to provide any new modules that may be specific to a club. It is also robust enough to support authentication like smart cards, and is designed to be secure and tamper proof with respect to the dates. Our clientele and product portfolio stand as testimony to this fact. Technology, vision and execution are the touchstones of our philosophy and what we stand by in our most challenging endeavours. This, coupled with our strong financial backing, allows us to deliver best in the class industry products.

Radio frequency identification (RFID) is a wireless technology that can be used to develop the access control system. This paper describes the design of RFID based security and access control system for use in bar and hostels inside the Club management system. The system combines RFID technology and bio-metrics to accomplish the required task. When the RFID reader installed at the entrance detects and read a smart card, the system scan the smart card and scans the database for a match. If both the card and database belong to a registered user, access is granted; otherwise the system turns on the alarm and makes an emergency call to the security van through GSM modem. In this way, the suspicious persons can be caught.

\section{A Review of Previous Research}

Peter Kieseberg, Manuel Leithner, Martin Mulazzani,[1] examines QR Codes and how they can be used to attack both human interaction and automated systems. As the encoded information is intended to be machine readable only, a human cannot distinguish between a valid and a maliciously manipulated QR code. Christoph Jechlitschek[2], provides a survey on radio frequency identification (RFID) technology. Initially RFID tags were developed to eventually replace barcodes in supply chains. Their advantages are that they can be read wirelessly and without line of sight, contain more information than barcodes, and are more robust. The paper describes the current technology, including the frequency ranges used and standards. With the increasing ubiquity of RFID tags, however, privacy became a concern. Roy Want[3], Many types of RFID exist, but at the highest level, we can divide RFID devices into two classes: active and passive. Active tags require a power source- - they're either connected to a powered infrastructure or use energy stored in an 
integrated battery. In the latter case, a tag's lifetime is limited by the stored energy, balanced against the number of read operations the device must undergo. One example of an active tag is the transponder attached to an aircraft that identifies its national origin. Another example is a Lo Jack device attached to a car, which incorporates cellular technology and a GPS to locate the car if stolen. Eben Upton[4] in UKbased Raspberry Pi Foundation at Cambridge University has begun manufacturing a $\$ 35$, creditcard sized computer, designed to make it affordable, bare-bones, tiny to teach basic computer science in schools worldwide. The Linux-based, single-board computer will have a $700-\mathrm{MHz}$ ARM architecture, CPU,256 M bytes of RAM, two USB ports and a 10/100 Ethernet controller. Shri Sant Gadge[5],Raspberry Pi is an innovative technology. The sheer number of users and fan base support the fact that the device can see an abundant future ahead. The device can certainly help anyone who really needs to learn electronics and computers. Raising the processing power can certainly assist the product in the future. Also supply a case and a appropriate instruction manual will get better the product.

\section{Proposed System}

Here the system uses RFID and integrated with a secure database to capture the entire fund utilization activities, proper receipt and bill generation facilities. This system is able to say how much money is left in the wallet of the organization, how much it could spend on different activities. The vat charges for the liquor products has been canceled from the government of Karnataka in the budget proposal 2017-18. It has capable to generate complete details of annual budget summary, members report, sales report, product reports, guest fee, death fund, card section report, income and expenditure reports, etc. The main entry gate and subsequent facility entrance doors are equipped with RFID readers. If the member is authorized and has adequate amount in the card, he/she can simply touch the card with RFID reader following which the access will be granted.

\subsection{RFID Detectors for smart card detection}

Smart Card Readers are used as a communications medium between the smart card and a host, such as a computer, POS Terminal or a mobile telephone. RFID belongs to a group of technologies referred to as Automatic Identification and Data Capture (AIDC). AIDC methods automatically identify objects, collect data about them, and enter those data directly into computer systems with little or no human intervention.

RFID methods utilize radio waves to accomplish this. At a simple level, RFID systems consist of three components: an RFID tag or smart label, an RFID reader, and an antenna. RFID tags contain an integrated circuit and an antenna, which are used to transmit data to the RFID reader (also called an interrogator). The reader then converts the radio waves to a more usable form of data. Information collected from the tags is then transferred through a communications interface to a host computer system, where the data can be stored in a database and analyzed at a later time.

\section{2-Objectives}

Objectives are the ones which describes the project outcome. To uphold the dignity of the individual. To honor all commitments. Commitment to quality, innovation and growth in every endeavor. Clients first: we value entrepreneurial, innovative and clientfocused attitudes. We deliver more value than expected. Professionalism: we are each personally accountable and jointly responsible for the success of the company and for the delivery of quality work to our clients. Integrity: we demand the highest levels of transparency and ethical behavior and personal integrity in all of our operations. Building for the future: we invest in our people and will empower them to realize their potential. Increase membership. Keep the management plan up to date. Promote squash at all levels from Junior to Senior. Keep the facilities modern. Develop full player activity programmes. Keep the club in a sound financial position.. Opportunities to increase skill levels of all players. Maintain good social activity., Develop a good family involvement. ,Develop a full computerized accounting package

\subsection{Developer Responsibilities}

Fixing system defects and Conversion of part of code $\&$ testing working on minor enhancements and major enhancements which will be treated as and Developments placed by clients. Response to inquiry, monitor the batch, support production run ,fix or solve application bugs and develop fixes for run time issues due to data errors. Meeting the SLAs for CMS like span, first time right $\&$ bug fixes to time resolution. 


\subsection{Mission}

Delivering the highest quality and most cost effective software products and services to our clients. Exceeding all client expectations. Creating a happy and challenging work environment. Continuously rewarding our stakeholders. Providing our clients with world-class expertise on a locally available basis. Assisting our clients in delivering lasting, differentiated and significant improvements in their performance whilst building a great firm that attracts, enhances and excites exceptional talent. To be the most innovative and dynamic organization, with the highest technical, financial and ethical performances.

\section{4-SYSTEM ARCHITECTURE}

\section{1- Modules}

A module is a small part of our project. This plays a very important role in the project and in coding concepts. In Software Engineering concept we treat it has a small part of a system but whereas in our programming language it is a small part of the program, which we also called as function in, some cases which constitute the main program.

The modules are

Admin Module:

Admin is the one who purchase the requested order from supplier and transfer of products from warehouse to different section. Admin has got all the privileges and overall control of the application.

Reception Module:

This module consists of member registration, luxury tax, room's facility, Guest fee, Death Fund. Once the member registered and get a membership card, he/she can allow to access all the facilities of the club depending on member type.

\section{> Bar Management:}

Sales of liquor and generates invoice of the daily sale and overall sales.

\section{Card Section:}

This section consist details about running games, closed games

\subsection{Architecture}

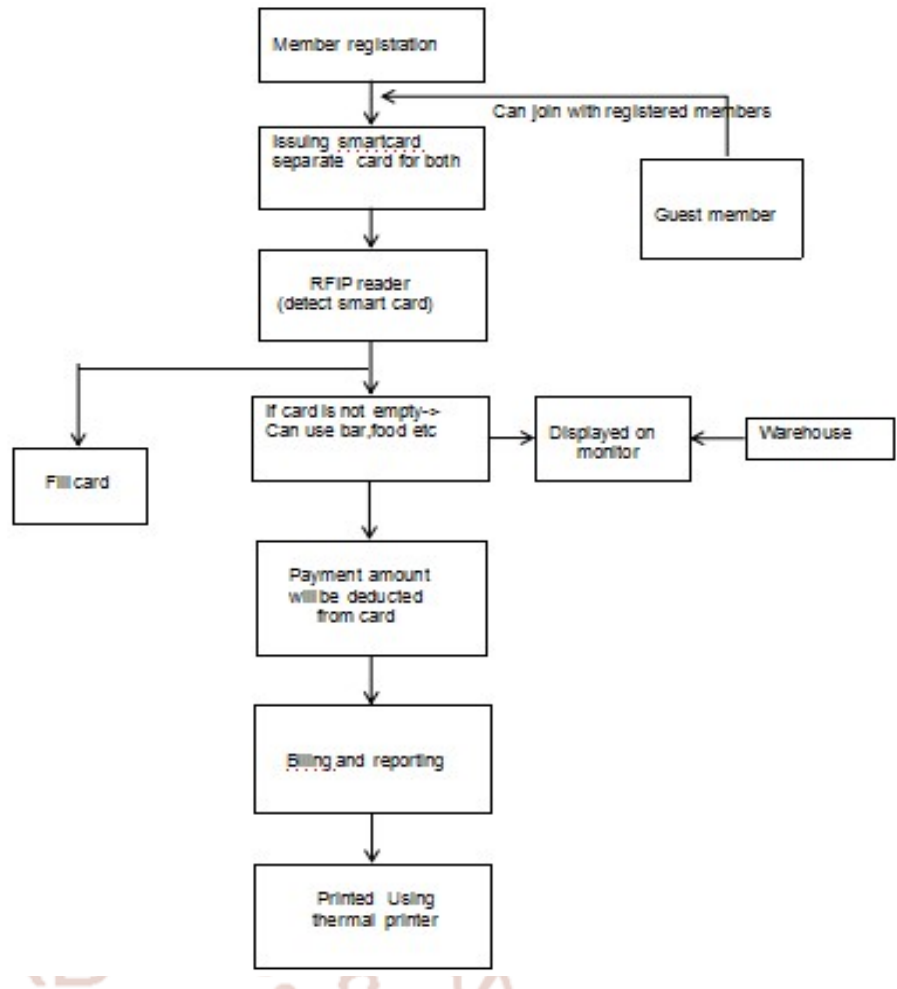

Fig 1: system architecture

\section{Work flow steps}

1. Member need to login before using any benefits in club if he registered before or else registration process will be takes-place.

2. After registration he will get a smart card using which he can login to the system.

3. Using that smart card he can use the benefits of the club after login successfully.

4. A RFID reader is used to detect the smart card which authenticates the truthfulness of customer..

5. Also the guest member can come with the members, they will be provided separate smart card which is valid for that particular time or one day.

6. Inside the club he can use the services like bar, food and other beverages And also he can play in card section only if his card have enough balance.

7. Every time he orders some food from the counter , amount in his card will be deducted. The order can be given from his club app or he can also send it through waiter. And this order will be displayed on the monitor screen which is kept in the kitchen section connected through raspberry pi.

8. The items to the bar section and kitchen section will be provided from the warehouse which maintains enough stocks for each section. 
9.In the bar section he can have any type of liquor and need to pay using his smart card..

10.Everyday the billing and the report will be made in the club. And if any kind of receipt has to be provided then it will be printed through thermal printer which uses less paper. every time the card is emptied the member can fill the amount $\mathrm{n}$ use it again .

\subsection{Some Advantages of Club Management System.}

There are few advantages in this application compared to older or existing club managing systems. Since we are using RFID detectors for the members activities the security has to be taken care. Some advantages of RFID in security are,

\section{Access Control}

Restrict and Permit Access using RFID based smart cards on a card by card basis RFID key cards only have to be placed close or within reasonable distance of a reader versus a swipe that may or may not register. More secure than cards with the magnetic strip, as they are embedded with an anti-cloning technology

\section{Asset Visibility}

Achieve full visibility of asset collections in real-time Streamline and fast-track asset collection audits Provides tight security by automatically tracking the movement of assets throughout location. If a piece moves beyond a preset boundary such as a room or doorway, security is instantly notified and the piece can be easily located.

Since the billing and reporting is done regularly in this system, the thermal printers are used for the billing purpose. Some important advantages of these are,

Increased Print Speed - Thermal printers are able to print at much faster rates than other printers. Their printing heads create images in milliseconds, resulting in much faster lines per second (lps) and images which dry extremely quickly.

Reduced Printing Costs - Thermal printers are inkless, using heat to react with the paper to create images, forgoing ribbons or cartridges in the process. The only consumable needed for thermal printing is the paper.

Improved Print Quality - Thermal printers create higher quality, more durable images than impact printers. They produce clear, long-lasting images that are more resistant to factors such as oils, climate, UV rays, etc. The images created by thermal printers are also more legible because there is no ink to smudge.

\section{CONCLUSION}

This project helps to keep a track of the customers, giving them the required benefits and recognizing customers at clubs will allow them to create an additional value as against their competition. In this project we are using a Radio Frequency Identification (RFID) and Thermal printers. Radio Frequency Identification (RFID) provides a smooth payment and secure access. Every club member has a unique RFID Smart card which contains details like name of the member, membership number, Flat number, contact details and photograph.

\section{REFERENCES}

1) Jerry Landt, "Shrouds of Time": outlines history and present of RFID: http://www.aimglobal.org /technologies/rfid/resources/shrouds_of_time.pdf

2) P. F. Baude, D. A. Ender, T. W. Kelley, M. A. Haase, D. V. Muyres, and S. D. Theiss, "Organic Semiconductor RFID Transponders", Electron Devices Meeting, 2003.

3) S. Inoue and H. Yasuura, "RFID privacy using user-controllable uniqueness", in Proc. RFID Privacy Workshop, Nov. 2003. http://www.rfidprivacy.us/2003/papers/sozo_inou e.pdf

4) "M. Feldhofer, S. Dominikus, and J. Wolkerstofer, "Strong Authentication for RFID Using the AES Algorithm", Cryptographic Hardware and Embedded Systems 2004. http://www.springerlink.com /openurl.asp?genre=article \&issn $=0302$ $9743 \&$ volume $=3156 \&$ spage $=357$

5) K. P. Fishkin, S. Roy, and B. Jiang, "Some methods for privacy in RFID communication", in Proc. 1st Eur. Workshop on Security in Ad-Hoc and Sensor Networks, 2004, http://www.intelresearch.net

/Publications/Seattle/062420041517_243.pdf

6) D. Haehnel, W. Burgard, D. Fox, K. Fishkin, and M. Philipose, "Mapping and Localization with WID Technology", International Conference on Robotics \& Automation, 2004. [Juels04] A. Juels, "Minimalistic Cryptography for Low-Cost RFID Tags", Security in Communication Networks 2004 [Krumm04] J. K 
7) Y.-P. Huang, Y.-T. Chang, and F. E. Sandnes.Ubiquitous information transfer across dierentplatforms by qr codes. J. Mobile Multimedia,6(1):3\{14, 2010.

8) ISO 16022:2006. Data Matrix bar code symbologyspeccation. ISO, Geneva, Switzerland.

9) ISO 18004:2006. QR Code bar code symbologyspecication. ISO, Geneva, Switzerland.

10) ISO 24778:2008. Aztec Code bar code symbology

11) specication. ISO, Geneva, Switzerland.

12) S. Lisa and G. Piersantelli. Use of $2 d$ barcode toaccess multimedia content and the web from a mobilehandset. In GLOBECOM, pages 5594\{5596, 2008. Member registration Issuing smartcard RFIP reader (detect smart card) Can use bar,foof etc Payment amount will be deducted from card Biling and reporting 